\title{
Un système en quête de succès
}

Le cas du Québec

In search of excellence. Vocational training in Quebec

Un sistema en búsqueda de éxito. El caso de Québec

Marc Tardif et André Balleux

\section{OpenEdition}

1 Journals

Édition électronique

URL : https://journals.openedition.org/ries/1592

DOI : 10.4000/ries.1592

ISSN : 2261-4265

Éditeur

France Education international

Édition imprimée

Date de publication : 1 décembre 2003

Pagination : 53-62

ISBN : 978-2-8542-0558-9

ISSN : $1254-4590$

Référence électronique

Marc Tardif et André Balleux, "Un système en quête de succès », Revue internationale d'éducation de Sèvres [En ligne], 34 | décembre 2003, mis en ligne le 22 novembre 2011, consulté le 09 juillet 2021. URL : http://journals.openedition.org/ries/1592 ; DOI : https://doi.org/10.4000/ries.1592

(c) Tous droits réservés 


\section{Un système en quête de succès}

\section{Le cas du Québec}

\section{Marc Tardif André Balleux}

La formation professionnelle initiale des jeunes au Canada n'est pas l'affaire d'un seul gouvernement. Le Canada est un pays composé de dix provinces, dont le Québec, et de trois territoires. La constitution du pays prévoit des champs de compétences centralisés et d'autres de juridiction provinciale dont l'éducation. Cette particularité canadienne implique que chaque province contrôle son système d'éducation de façon indépendante. "Au sein de la fédération canadienne, ce sont les gouvernements des provinces qui assument l'entière responsabilité du développement de l'éducation et de la formation sur leur territoire " ${ }^{1}$. Par conséquent, il n'y a pas un système éducatif unique au Canada et il s'avérerait hasardeux de tenter une généralisation, au niveau du pays, des structures, finalités, objectifs et programmes de formation professionnelle initiale des jeunes des provinces prises séparément. Une seconde caractéristique importante concerne la langue d'enseignement. Le Canada est un pays qui compte deux langues officielles, le français et l'anglais. Au Québec, les réseaux d'enseignement francophone et anglophone cohabitent, le premier étant majoritaire, et sont régis par le ministère de l'Éducation du Québec. Les informations contenues dans cet article révèlent la situation vécue au Québec tant pour la population francophone qu'anglophone.

L'histoire récente de la formation professionnelle est marquée par deux réformes. Pour être en mesure de comprendre adéquatement la dernière réforme majeure, celle de 1986, il est nécessaire de connaître le contexte et les raisons qui ont mené à ces changements.

\section{LA RÉFORME DE 1964}

Au Québec, depuis le milieu des années soixante, la formation initiale des jeunes est placée sous la responsabilité du ministère de l'Éducation du Québec (MEQ) qui vient d'être créé. C'est à ce moment qu'est décidée la création d'écoles de formation professionnelle, appelées jusqu'alors des écoles d'arts et métiers, intégrées dans de nouvelles institutions de formation appelées écoles polyvalentes. Assez rapidement, cette nouvelle cohabitation de la formation

1. Ministère de l’Éducation du Québec, 2002, p. 32. 
professionnelle et de la formation générale sous un même toit, celui des écoles polyvalentes, entraîne des effets négatifs marqués. Charland (1982) indique qu'au début des années soixante-dix, des voix se font entendre pour souligner que, de façon générale dans les écoles polyvalentes, la formation professionnelle est dévalorisée par rapport à la formation générale. Des préjugés tenaces associent les élèves de la formation professionnelle à des élèves connaissant des difficultés d'apprentissage. Sont aussi dénoncés les équipements désuets et insuffisants dans les ateliers, le manque d'enseignants compétents et la difficulté pour les élèves inscrits dans les programmes de formation professionnelle de revenir à la formation générale ou de poursuivre leurs études à des niveaux supérieurs. Enfin, la formation professionnelle est considérée comme un cul-desac pour les élèves qui s'y inscrivent.

Le nombre d'inscriptions aux programmes de la formation professionnelle dans les écoles polyvalentes, programmes alors exclusivement réservés aux jeunes en formation initiale, atteint un maximum en 1976-1977. Par la suite, c'est le déclin. "Avant la réforme, une tendance est claire : l'effectif des jeunes en formation professionnelle est nettement en baisse. De 1977-1978 à 1986-1987, il diminue de $59 \%$, passant de 105944 à 43747 élèves » ${ }^{2}$.

\section{LA RÉFORME DE 1986}

C'est en 1986 qu'est annoncée une restructuration majeure de la formation professionnelle au Québec; elle sera mise en œuvre l'année suivante. Elle vise à «accroître la qualité de la formation professionnelle ainsi qu'à assurer une meilleure adéquation avec les besoins du marché du travail ${ }^{3}$.

Bien que ces écrits récents du ministère de l'Éducation du Québec attirent l'attention sur les objectifs de qualité de la formation et d'adéquation avec le marché du travail, il n'en demeure pas moins qu'un autre objectif majeur était présent lors de cette réforme, ce qui est confirmé par le Conseil supérieur de l'éducation (1992) qui indique que, la diminution des effectifs étant particulièrement inquiétante, l'un des objectifs majeurs est «la revalorisation de la formation professionnelle ${ }^{4}$.

L'accessibilité des jeunes, puisque c'est bien d'eux dont il est question à ce moment, à la formation professionnelle initiale, était au cœur de la réforme de 1986. Toutefois, il apparaît évident que cette réforme implantée en 1987 n'a pas eu l'effet escompté auprès des jeunes. De 28604 jeunes inscrits en formation professionnelle initiale en 1987-1988, ils ne sont plus que 7537 en 1993-1994 ${ }^{5}$.

\footnotetext{
2. Ministère de l'Éducation du Québec, 1999, p. 2.

3. Ministère de l'Éducation du Québec, 2002, p. 27.

4. Conseil supérieur de l'éducation, 1992, p. 10.

5. Ministère de l'Éducation du Québec, 1999.
} 
Il faut noter ici un autre changement important lié à la formation professionnelle. Au cours des années quatre-vingts, l'idée de permettre aux adultes l'accès au système scolaire de façon générale, et par conséquent aux programmes d'enseignement professionnel, fait son chemin. Bien reçue par les milieux concernés par la formation professionnelle, cette idée fait modifier la Loi sur l'instruction publique en ce sens en 1988. Dès lors, les élèves adultes sont intégrés dans les programmes de formation professionnelle. L'intégration est totale puisque les groupes d'élèves sont composés de jeunes et d'adultes qui suivent les mêmes formations. Depuis, on retrouve des statistiques d'effectifs scolaires pour les moins de vingt ans et pour les vingt ans et plus. Ainsi, il ne faut pas confondre les moins de vingt ans, qui peuvent avoir un statut d'adulte, avec les jeunes en formation professionnelle initiale. La distinction est importante puisqu'en 1993-1994, on compte 7537 jeunes en formation professionnelle initiale comparativement à 18480 jeunes de moins de vingt ans inscrits à un programme de formation professionnelle.

Au milieu des années quatre-vingt-dix, la préoccupation de la fréquentation des jeunes dans les programmes de formation professionnelle est encore présente. En 1995, le groupe de travail sur la relance de la formation professionnelle des jeunes au secondaire et de la formation technique indique que "le défi le plus considérable que le système de formation professionnelle doit relever est celui d'attirer les jeunes en continuité de formation » ${ }^{6}$. Ce groupe de travail propose le défi suivant : "d'ici l'an 2000, multiplier par au moins quatre le nombre de diplômes de formation professionnelle décernés à des jeunes de moins de vingt ans ${ }^{7}$.

L'année suivante, la commission des états généraux sur l'éducation (ministère de l'Éducation du Québec, 1996), se référant à l'idée qu'aucun jeune au Québec ne devrait quitter le système scolaire sans y avoir acquis une qualification professionnelle, précise : «il faut reconnaître que la réforme de la formation professionnelle, en particulier depuis 1986, nous a éloignés de cet objectif. En effet, dans l'espoir de revaloriser cette filière, que l'on considérait trop associée aux élèves éprouvant des difficultés, on en a haussé les exigences d'entrée et reporté l'accès de deux ans, soit après la $5^{\mathrm{e}}$ secondaire. Même si par la suite ces exigences ont été revues à la baisse, force est de constater que cette réforme, loin d'attirer plus de jeunes en formation professionnelle, les en a plutôt éloignés. » ${ }^{8}$

\footnotetext{
6. Groupe de travail, 1995, p. 40.

7. Ibid., p. 40.

8. Ministère de l’Éducation du Québec, 1996, p. 77.
} 


\section{UN BILAN RÉSERVÉ}

Concernant le défi posé par le groupe de travail sur la relance de la formation professionnelle des jeunes au secondaire et de la formation technique, de toute évidence, il n'a pas été relevé. Selon les données recueillies (Beaudet, 2002), en 1994-1995, 4655 diplômes étaient émis aux moins de vingt ans et ce nombre est passé à 8674 en 1999-2000, soit un peu moins du double, ce qui nous laisse loin du compte des aspirations prévues.

Encore tout récemment, le Conseil supérieur de l'éducation notait dans son rapport annuel de 2002 «la faible présence des jeunes dans les programmes de formation professionnelle ${ }^{9}$. La situation n'a pas vraiment évolué chez cette clientèle des jeunes de moins de vingt ans.

Si la situation est problématique pour les jeunes de moins de vingt ans en formation professionnelle, et plus encore pour les jeunes en formation professionnelle initiale, les divers changements apportés au système de la formation professionnelle au Québec en 1986 et depuis cette date importante ont certainement favorisé la présence des adultes dans ces programmes.

\section{Motifs favorisant l'accès des adultes à la formation professionnelle}

L'ensemble des motifs présentés ici vise à montrer comment le système d'enseignement professionnel au Québec s'est développé selon la logique de l'éducation des adultes.

La réforme de la formation professionnelle en 1986 a été une première étape menant vers un système plus attrayant pour les adultes que pour les jeunes. Entre autres pour se défaire de l'image négative des filières de formation auxquelles était associée celle des élèves en difficultés, on a exigé que les jeunes se destinant à la formation professionnelle possèdent une solide formation de base : les exigences d'entrée à l'enseignement professionnel étaient haussées. Cette hausse des exigences d'entrée, bien entendu en plus de bien d'autres changements, devait motiver les jeunes à s'inscrire dans les programmes de formation professionnelle. La réalité a été contraire aux objectifs et a mis en péril tout le système de l'enseignement professionnel. C'est une modification de la Loi sur l'instruction publique en 1988, qui ouvre la porte à l'élève adulte dans les programmes de formation professionnelle, qui permet au système de survivre. Depuis, les inscriptions des adultes en formation professionnelle ont été, année après année, nettement supérieures à celles des jeunes en formation initiale.

9. Conseil supérieur de l'éducation, 2002, p. 24. 
L'analyse des changements survenus au cours des années qui suivirent a permis au groupe de travail sur la relance de la formation professionnelle des jeunes au secondaire et de la formation technique (1995), sur cette question précise des adultes en formation professionnelle, d'indiquer que «le dispositif actuel de formation professionnelle semble répondre plus adéquatement aux besoins de formation des adultes qui ont besoin de se donner une formation spécialisée, d'une façon intensive, les préparant à occuper un emploi rapidement ${ }^{10}$.

En 1997, le groupe de travail sur la réforme du curriculum associe la dimension sociale à son analyse et en arrive à une conclusion semblable. "La demande sociale pour des études plus longues a entraîné une diminution forte du nombre d'élèves inscrits en enseignement professionnel. Ce fait, auquel s'ajoute la nécessaire modernisation des entreprises, a conduit, voici dix ans, à une réforme déplaçant plus haut dans le parcours scolaire, le niveau où la formation professionnelle devait dorénavant se donner. [...] L'effet de système de cette réforme a été le suivant : l'enseignement professionnel est surtout fréquenté par les adultes. ${ }^{11}$

Encore plus, cette même année, la Loi sur l'instruction publique a une fois de plus été modifiée, donnant ainsi un régime pédagogique à l'enseignement professionnel et surtout précisant les conditions de gratuité des services éducatifs pour les élèves adultes. En 2002, malgré l'observation d'une hausse de l'effectif scolaire en formation professionnelle et du nombre de diplômes émis en formation professionnelle depuis presque dix ans, le Conseil supérieur de l'éducation du Québec est sans équivoque. "Il importe de souligner la faible présence des jeunes dans les programmes de formation professionnelle ${ }^{12}$.

Pour sa part, Beaudet (2002) indique, dans la conclusion de son bilan de la réforme de l'enseignement professionnel, à titre d'exemple pour appuyer le constat de l'approche marché du travail du système de l'enseignement professionnel, «un cadre réglementaire et financier et des pratiques de sélection qui favorisent les élèves les plus âgés, les plus matures et les plus motivés à s'insérer ou à se réinsérer dans le marché de l'emploi » ${ }^{13}$. Il conclut ainsi : «la réforme de l'enseignement professionnel, pourtant voulue pour rehausser le nombre de jeunes en ce secteur, s'est éloignée de cet objectif [...] en formant, selon un modèle standardisé de haut niveau, le plus grand nombre possible de personnes parmi les plus aptes et les plus prêtes à accéder au marché du travail ou à y retourner, en l'occurrence les adultes ou ceux ayant fait un détour par le collégial. ${ }^{14}$

\footnotetext{
10. Groupe de travail, 1995, p. 24.

11. Groupe de travail, 1997, p. 38.

12. Conseil supérieur de l'éducation, 2002, p. 25.

13. A. Baudet, 2002, p. 37.

14. Ibid., p. 38.
} 


\section{Motifs décourageant l'accès des jeunes à la formation professionnelle initiale}

Bien que l'enseignement professionnel au Québec soit de plus en plus valorisé et reconnu, certains motifs découragent ou, à tout le moins, n'encouragent pas les jeunes à s'y inscrire en formation initiale.

Même s’il est vrai que l'enseignement professionnel au Québec a connu un très important déficit d'image à partir des années soixante-dix, la situation s'est grandement améliorée au cours des dernières années. Les employeurs affichent un haut taux de satisfaction concernant la qualité de la formation des diplômés de la formation professionnelle ${ }^{15}$, comme le constate en 2002 le Conseil supérieur de l'éducation.

Beaudet (2002) constate au début de son bilan de la réforme de l'enseignement professionnel que «le système de formation professionnelle a su relever le défi de la qualité, bénéficie désormais d'une image de marque auprès de ceux qui la connaissent et dégage un sentiment de fierté de plus en plus réel auprès de ceux qui y œuvrent ». Enfin, en ce qui concerne l'information relative à la formation professionnelle et la perception au sujet de cette filière, il ajoute : «mais la meilleure information qui circule est celle des élèves formés et de leurs parents, des employeurs qui les ont reçus en stage ou qui les emploient, celle enfin des enseignantes et enseignants fiers d'œuvrer en formation professionnelle. L'enseignement professionnel, on peut le souligner, n'a plus la réputation qui était sienne au moment de la réforme. Mais l'information ne circule pas encore très bien, et, doit-on le dire, particulièrement auprès des enseignants (sic) du primaire et du premier cycle du secondaire. Toutefois, les parents apparaissent de moins en moins mal à l'aise de voir leurs enfants sinon, ceux du voisin, se diriger vers l'enseignement professionnel. C'est probablement le principal gain de la réforme ${ }^{16}$.

Cette revalorisation de l'enseignement professionnel est toutefois accompagnée de freins à l'accès des jeunes en formation initiale. Il appert que l'objectif d'une meilleure adéquation de la formation avec les exigences du marché du travail a supplanté l'objectif d'accessibilité des jeunes. "Le système québécois de formation professionnelle et technique (FPT) et son ingénierie s'appuient, rappelons-le, sur quelques prémisses fondamentales correspondant à des choix politiques de la société québécoise. La FPT est définie en étroite collaboration avec le marché du travail. Les compétences requises pour exercer les fonctions de travail sont au cœur de la démarche de développement des programmes (système piloté par la demande). La mise en place et la gestion du

15. Gouvernement du Québec, 2000; 2003.

16. Ibid., p. 35 . 
système sont effectuées en étroit partenariat avec les acteurs intéressés, en particulier les représentants et les représentantes du marché du travail ${ }^{17}$.

Beaudet (2002) confirme ce déplacement en indiquant que «la réforme de l'enseignement professionnel, pourtant voulue pour rehausser le nombre de jeunes en ce secteur, s'est éloignée de cet objectif et s'est largement centrée sur l'objectif de répondre aux besoins du marché du travail» ${ }^{18}$.

\section{Une diversification précoce des voies de formation}

La réforme de 1986 était nécessaire pour détacher les filières de l'enseignement professionnel de l'image négative qui lui était accolée. Pour y arriver, les exigences d'entrée avaient été rehaussées. En référant au nouveau programme proposé, le ministre de l'Éducation d'alors affirmait que «pour les élèves âgés de douze à seize ans qui ne peuvent pour diverses raisons suivre les cheminements réguliers de formation, des cheminements particuliers seront aménagés afin d'accroître le plus possible leur formation de base ${ }^{19}$.

Toutefois, ce n'est qu'en 1995 que le groupe de travail sur la relance de la formation professionnelle des jeunes au secondaire et de la formation technique recommande une diversification, de façon expérimentale, de voies de formation. Ainsi, différentes voies de formation sont implantées. «L'une d'entre elles mène à l'obtention d'une attestation de formation professionnelle (AFP) donnant accès à l'exercice de métiers semi-spécialisés. Cette voie de formation a pour objectifs de contrer le décrochage scolaire et de répondre aux besoins locaux de maind'œuvre. L'élève acquiert sa formation professionnelle en entreprise sous la supervision d'un "travailleur-parrain». [...] Une autre voie permet à des élèves des classes de $4^{\mathrm{e}}$ et $5^{\mathrm{e}}$ secondaire en formation générale, de profiter d'une année d'exploration professionnelle. [...] La voie de la concomitance permet aux élèves de s'inscrire à un programme de formation professionnelle même s'ils n'ont pas encore obtenu les unités de $4^{\mathrm{e}}$ ou de $5^{\mathrm{e}}$ secondaire requises pour y accéder. Ils doivent toutefois accumuler ces unités durant leur formation professionnelle afin d'obtenir leur diplôme d'études professionnelles » 20 .

Ces volets ont été mis à l'essai dans plusieurs établissements d'enseignement sur le territoire québécois. Deux ans après cette implantation expérimentale, le groupe de travail sur la réforme du curriculum (1997) se prononce sur la diversification des voies de formation professionnelle. «En 1995-1996, le ministère de l'Éducation débutait l'implantation d'un programme expérimental de diversification des voies en formation professionnelle. Un des objectifs de ce

\footnotetext{
17. Ministère de l'Éducation du Québec (2002) p. 30.

18. p. 38 .

19. Ryan, 1986, p. 5 .

20. Gouvernement du Québec, 2003, p. 9.
} 
programme est d'offrir aux jeunes, en plus de la formation professionnelle intensive déjà existante, des choix diversifiés de formation préparatoire au marché du travail qui puissent débuter avant la fin du secondaire. Deux volets de ce programme, la formation aux métiers semi-spécialisés et la formation en concomitance, nous apparaissent répondre efficacement au besoin de diversification des voies évoquées plus haut. Nous proposons donc que la mise en place de ces deux volets s'intensifie et se généralise afin que tous les élèves puissent choisir l'option qui corresponde à leurs besoins sans être obligés de compléter des études qu'ils jugent souvent trop longues.»

Toutefois, en 2002, le Conseil supérieur de l'éducation conclut que «la réforme tarde ou se révèle encore incomplète sur le plan de la diversification des voies de formation au secondaire, incluant la formation professionnelle».

Enfin, sur cette question de la diversification des voies de formation professionnelle, le Conseil supérieur de l'éducation (2002) «s'étonne d'ailleurs que sept ans après la mise en ouvre du programme expérimental de diversification des voies offertes aux jeunes en formation professionnelle, aucun bilan officiel n'ait été publié».

\section{L’apprentissage}

Malgré une longue tradition, les systèmes d'apprentissage basés sur

l'expertise des milieux de travail ont périclité pour ne présenter aujourd'hui que des filières de formation très marginales (Balleux, 2000). Dépendant du ministère de l'Emploi, ces filières ne conduisent pas à une formation qualifiante. Une tentative de collaboration entre le MEQ et le ministère de l'Emploi (1997-1999) aurait pu mettre en place un système en alternance et provoquer ainsi une revalorisation de la formation en apprentissage. Malheureusement, la formule a été implantée trop rapidement, sans le support nécessaire, et abandonnée depuis.

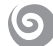

Dans le deuxième chapitre de son rapport annuel sur l'état et les besoins de l'éducation au Québec, le Conseil supérieur de l'éducation (2002) trace les grandes orientations et précise des objectifs précis à atteindre au cours des prochaines années. À l'intérieur de la première orientation, qui est d'accroître la réussite scolaire des jeunes telle qu'attestée par l'obtention d'un premier diplôme d'études secondaires avant l'âge de vingt ans, tout un axe d'interventions est dédié à la formation professionnelle.

Intitulé «le développement d'une architecture multiforme et intégrée de formation professionnelle pour les jeunes ${ }^{21}$, cet axe comporte quatre principes que devrait respecter cette architecture souple : un réel accès à une for-

21. Conseil supérieur de l'éducation, 2002, p. 53. 
mation professionnelle valorisante pour les jeunes; l'articulation des filières de formation et l'élimination des impasses; la diversification des filières et des formes de formation pratique; une préoccupation particulière pour les besoins des élèves de seize à dix-huit ans.

Ces quatre principes révèlent bien les difficultés du système actuel au regard de la formation professionnelle initiale des jeunes au Québec. Les modifications à apporter n'imposent pas de renier tous les acquis du système actuel mais visent surtout à remédier à une faiblesse importante qui s'est accentuée avec les années: le désintérêt des jeunes pour la formation professionnelle initiale.

\section{BibLIOGRAPHIE}

BALLEUX A. (2000) : "Les filières de formation professionnelle par apprentissage au Québec : état de la situation et enjeux de l'évolution actuelle». La Revue canadienne pour l'étude de l'éducation des adultes, 14 (2), pp. 51-74.

BEAUDET A. (2002): La réforme de l'enseignement professionnel : bilan et perspectives. Québec : Association des cadres scolaires du Québec.

CHARLAND J.-P. (1982): Histoire de l'enseignement technique et professionnel. Québec : Institut québécois de recherche sur la culture.

Conseil supérieur de l'éducation (1992): En formation professionnelle: L'heure d'un développement intégré. Sainte-Foy : Direction des communications du Conseil supérieur de l'éducation.

Conseil supérieur de l'éducation (2002): Rapport annuel sur l'état et les besoins de l'éducation. La gouverne de l'éducation, priorités pour les prochaines années. Sainte-Foy : Direction des communications du Conseil supérieur de l'éducation.

Gouvernement du Québec (2000): La formation professionnelle au secondaire: les employeurs s'expriment. Québec : Ministère de l'Éducation.

Gouvernement du Québec (2003): La formation professionnelle et technique au Québec : un aperçu. Québec : Ministère de l'Éducation.

Groupe de travail sur la réforme du curriculum (1997): Réaffirmer l'école. Prendre le virage du succès. Québec: Gouvernement du Québec, Ministère de l'Éducation du Québec.

Groupe de travail sur la relance de la formation professionnelle des jeunes au secondaire et de la formation technique (1995): La formation professionnelle chez les jeunes : un défi à relever. Québec : Ministère de l'Éducation du Québec.

Ministère de l'Éducation du Québec (1986): Plan d'action, la formation professionnelle au secondaire. Québec: Gouvernement du Québec. 
Ministère de l'Éducation du Québec (1996): Les États généraux sur l'éducation. 1995-1996. Exposé de la situation. Québec: Gouvernement du Québec.

Ministère de l'Éducation du Québec (1999): Bulletin statistique de l'éducation, $n^{\circ}$ 12. Québec: Gouvernement du Québec, Direction des statistiques et des études quantitatives.

Ministère de l'Éducation du Québec (2002): La formation professionnelle et technique au Québec. Un système intégrant l'ingénierie de gestion et l'ingénierie de formation. Québec: Gouvernement du Québec, ministère de l'Éducation, secteur de la formation professionnelle et technique et de la formation continue, direction générale des programmes et du développement. 